\title{
Quantification of Palbociclib in Spiked Human Plasma Using Liquid Chromatography- Electro Spray lonization-tandem Mass Spectrophotometry- an Application to Pharmacokinetic Study
}

\author{
Badikela Ramakrishna ${ }^{1}$, Sumanta Mondal ${ }^{1, *}$, Prasenjit Mondal ${ }^{2}$ \\ 'Department of Pharmaceutical chemistry, GITAM Institute of Pharmacy, GITAM (Deemed to be University), Gandhi Nagar, Rushikonda, Visakhapatnam, \\ Andhra Pradesh, INDIA. \\ ${ }^{2}$ Vaageswari Institute of Pharmaceutical Sciences, Karimnagar, Telangana, INDIA.
}

\begin{abstract}
Objectives: The present method was developed for the estimation of palbociclib in spiked human plasma using Liquid chromatographic mass spectroscopy. Methods: The liquid-liquid extraction method was adopted and chromatographic separation was performed on a waters symmetry shield, $C_{18}(4.6 \mathrm{~mm}$ id $\times 50 \mathrm{~mm}$ ) analytical column using (methanol: ultrapure water, $\mathrm{pH} 4.2$ in the volume ratio of $60: 40$ ) as mobile phase. Positive ion mode was selected to obtain the product $\mathrm{m} / \mathrm{z}+447.5$ (parent) $\rightarrow 380.3$ (product) for palbociclib and $\mathrm{m} / \mathrm{z}+434.5$ (parent) $\rightarrow 322.7$ (product) for internal standard. Results: Calibration curve was linear over the range of $3-600 \mathrm{ng} / \mathrm{ml}$. The intra and interday accuracy with \% nominal $95 \rightarrow 98.4 \%$, precision $\% \mathrm{CV} \leq 2 \%$ in all quality control levels, The percentage extraction recovery $(96.15 \% \rightarrow 98.34 \%)$, demonstrated excellent matrix and analyte selectivity ( $\%$ interference $=0$ ), and satisfactory stability study results in all types (\% nominal $93.91 \% \rightarrow 99.58 \%$ ). The pharmacokinetic parameters was studied in the rabbit model, and the area under the curve
\end{abstract}

$\left(\right.$ AUC $_{0-\infty}$ ) for palbociclib was $2632 \pm 6.98 \mathrm{hr} . \mathrm{ng} / \mathrm{ml}$. The elimination half-life $\left(\mathrm{t}_{1 / 2}\right)$ is $11.3 \pm 5.3 \mathrm{hr}$. Conclusion: Based on the experimental findings the current developed method was considered a novel validated bioanalytical method, and applied in rabbit blood samples for pharmacokinetic studies of marketed formulations.

Key words: Palbociclib, Bioanalytical, Pharmacokinetic study, LCMS/MS, method development.

Correspondence

Dr. Sumanta Mondal,

Associate professor and NSS Programme Officer (Unit-IX), GITAM Institute of Pharmacy, GITAM (Deemed to be University), Visakhapatnam-530045, Andhra Pradesh, INDIA.

Email id: mondalresearch@gmail.com

DOI: 10.5530/jyp.2022.14.16

\section{INRODUCTION}

Breast cancer $(\mathrm{BC})$ is the most common cancer in women and the second most common cancer overall. Despite being treatable, metastatic breast cancer (MBC) is still a nearly incurable condition, with a median overall survival of just three years ${ }^{1}$ and a 5 -year OS of just $25 \%$. Palbociclib, ribociclib, and abemaciclib ${ }^{2}$ have recently been approved for the treatment of endocrine-resistant $\mathrm{MBC}$ in conjunction with ET due to their effectiveness in extending progression-free survival, increasing clinical benefit rate (CBR), and the response rate in various clinical contexts and treatment lines. ${ }^{3}$ Palbociclib which is chemically [6-Acetyl8-cyclopentyl-5-methyl-2-\{[5-(piperazin- 1-yl) pyrindin-2-1] amino $\}$ pyrido [2, 3-d]-pyrimidin-7(8H)-one] Pfizer has developed Palbociclib (Figure 1), a drug for the treatment of ER-positive and HER2(Human epidermal growth factor receptor 2)-negative breast cancer., ${ }^{4,5}$ Given the importance of palbociclib, a simple, validated method for estimating plaboiclib in human plasma is needed, allowing for the estimation of a very low plaboiclib concentration in blood. But the extensive literature review revealed that there is an availability of few HPLC work ${ }^{6-9}$ for the determination of palbociclib alone or in combination with other anticancer agents. There is an availability of LC-MS/MS methods, ${ }^{10-12}$ for the simultaneous estimation of palbociclib with other anticancer agents like abemaciclib, ribociclib, letrozole, carbozantinib, olapertib, pazopanib, sorafenib and sunitinib. But no such LCMS-MS methods was reported for the palbociclib alone in human plasma and its kinetic study. Though one HPLC methods ${ }^{13}$ reported about the determination of palbociclib alone in plasma samples but have several drawbacks e.g. The concentration of analyte was not in nanogram level, which indicated low sensitivity. More baseline noise is seen in the chromatograms given. They utilized solid phase extraction, which is an expensive factor and has poor recovery. Very few stability studies have been conducted in that documented method, which cannot be sufficient to support stability as per USFDA guidelines. The investigator did not carry out substantial validation parameters, such as dilution integrity, matrix effect and robustness. Efforts were therefore made with the objective to rectify the recorded disadvantages and to develop and validate a novel sensitive LCMS/MS method for palbociclib alone in human plasma as per USFDA guidelines ${ }^{14}$ and to apply the method developed for the pharmacokinetic analysis using rabbit plasma samples.

\section{MATERIALS AND METHODS}

Palbociclib standard (99.95\% pure) and ribociclib (99.89\% pure) was kindly provided by Hetero drugs limited, Hyderabad, India, as a gift sample. Ammonium format buffer was obtained from Sigma- Aldrich, Hyderabad, India. HPLC grade water was procured form Millipore mili-Q.

\section{High Performance Liquid chromatographic operating conditions}

An isocratic elution technique was adopted with the mobile phase (methanol: ultrapure water, $\mathrm{pH} 4.2$ in the volume ratio of 60:40) derived at a flow rate of $600 \mu \mathrm{l} /$ minute using waters Symmetry shield RP Ccolumn with $4.6 \mathrm{~mm}$ internal diameter, $5 \mu \mathrm{m}$ particle size, $100 \mathrm{~A}^{0}$ pore size. 


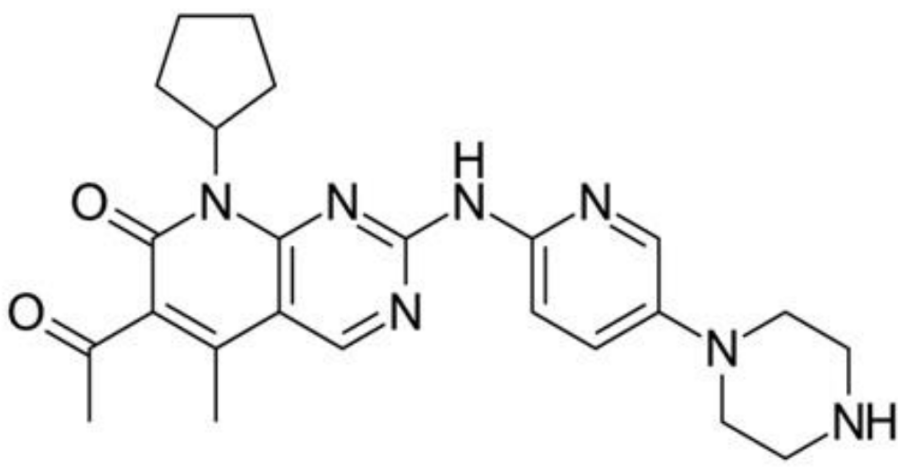

Figure 1: Chemical structure of Palbociclib.

\section{Mass spectrometry operating conditions}

Mass spectrometry was performed using an API-3000 triple quadrupole mass spectrometer (AB SCIEX, Foster city, CA/concord, Ontario, Canada) was equipped with an electrospray ionisation source (ESI), operating in the positive ion mode at $700^{\circ} \mathrm{C}$ dessolvation temperature. The ion source voltage was $5000 \mathrm{~V}$, the source temperature was maintained $382^{\circ} \mathrm{C}$. The entrance potential and collision energy was maintained $10 \mathrm{~V}$ and $38 \mathrm{~V}$. All other tuning parameters was set for the palbociclib and internal standard Ribociclib. Detection of the ions were carried out in multiple reaction monitoring by monitoring the transition pairs of $\mathrm{m} / \mathrm{z}+447.5$ (parent) $\rightarrow 380.3$ (product) for palbociclib and $\mathrm{m} / \mathrm{z}$ +434.5 (parent) $\rightarrow 322.7$ (product) for internal standard.

\section{Preparation of calibration curve and quality control sample}

The calibration curve samples and quality control samples of palbociclib was prepared form the standard aqueous stock solution of $1 \mathrm{mg} / \mathrm{mL}$. Further dilution was made and finally plasma spiked calibration curve samples was prepared in the range of 0.3 to $600 \mathrm{ng} / \mathrm{mL}$. Quality control samples of palbociclib was prepared by diluting standard stock solution $(1 \mathrm{mg} / \mathrm{mL})$ in the range of 1000 to $10 \mathrm{ng} / \mathrm{mL}$ of aqueous solutions. Then plasma spiked quality control samples was prepared by the mixing a definite volume of human plasma to the diluted solutions to obtained the concentrations in the range of $600 \mathrm{ng} / \mathrm{mL}$ (DIQC), $300 \mathrm{ng} / \mathrm{mL}$ (HQC), $100 \mathrm{ng} / \mathrm{mL}($ MQC-II), $50 \mathrm{ng} / \mathrm{mL}(\mathrm{MQC}-\mathrm{I}), 10 \mathrm{ng} / \mathrm{mL}(\mathrm{LQC}), 0.3 \mathrm{ng} / \mathrm{mL}$ (LLOQ) level.

\section{Preparation of mobile phase}

For the preparation of $100 \mathrm{~mL}$ of mobile phase, $60 \mathrm{~mL}$ of HPLC grade methanol was transferred in to clean and dry volumetric flask. $40 \mathrm{~mL}$ of ultrapure water $\left(\mathrm{P}^{\mathrm{H}} 4.2\right)$ was slowly added to the flask to achieve the final solution of mobile phase (Methanol: Ultrapure water, 60:40).

\section{Sample preparation}

A simple liquid-liquid extraction method was developed for sample processing. For the calibration curve and quality control samples, the palbociclib plasma samples and the internal standard (ribociclib) were thawed at room temperature. Vortexed the samples to ensure full blending. With the exception of blank plasma samples, where only $25 \mu \mathrm{l}$ of diluent (mobile phase) was added, $250 \mu \mathrm{l}$ of palbociclib plasma sample and Ribociclib internal standard were pipetted out and placed in $15 \mathrm{ml}$ of Stoppard tubing. Tetra butyl methyl ether $(5 \mathrm{~mL})$ was used as an extraction solvent, and each tube was shaken at $200 \mathrm{rpm}$ for $20 \mathrm{~min}$ on a reciprocating shaker. The samples were centrifuged at $4000 \mathrm{rpm}$ for $10 \mathrm{~min}$ at room temperature. Under nitrogen gas, the organic supernatant layer was transferred to pre-labeled tubes and evaporated to dryness at $40^{\circ} \mathrm{C}$. Both prepared samples were reconstituted with $200 \mu \mathrm{l}$ of mobile procedure, transferred to autosampler loading vials, and $10 \mu \mathrm{l}$ of the sample was injected into the LC-MS/MS device.

\section{Method validation \\ Carry over effect}

The autosampler was used to monitor the carryover effect of palbociclib and the internal standard (ribociclib). Six replicates of analytes and internal reference samples were analysed at the null, ULOQ, and LLOQ levels. The processed samples were inserted in a particular order, for example, LLOQ samples first, then ULOQ, then blank. At the retention of analytes and IS, the interfering peak response of blank samples should not exceed $5 \%$ of the average IS response.

\section{Matrix effect}

This research was done at the LQC and HQC levels. For this analysis, one haemolytic and one lipemic plasma lot were chosen. One set of each sample was spiked with blank matrices (haemolytic), while the other set (LQC and HQC) with internal standard was spiked with lipemic plasma. Six replicates of aqueous samples with final LQC and HQC concentrations were prepared by adding an internal standard to reconstituted palbociclib and injecting each one separately. By dividing peak response area in the presence of matrix ion by mean peak area response ratio in the absence of matrix ion, an internal standard normalised matrix factor was determined. The IS-normalized factor's variability, as determined by the coefficient of variance, should be less than $15 \%$.

\section{Precision and accuracy}

Using several palbociclib QC samples at the level of LLOQ, LQC, MQC(I and II), and HQC in six replicates, the precision (intra and interday) and accuracy were estimated, and the concentrations in these levels were measured, followed by standard deviation, percent $\mathrm{CV}$ for precision, and percent nominal for accuracy for each replicate. The acceptance requirements for accuracy (percent nominal) are $15 \%$ and $20 \%$ for LLOQ level, respectively, and precision (percent CV) should be within $15 \%$ and $20 \%$ for LLOQ sample, respectively.

\section{Linearity}

The linearity of the current approach was also tested in the palbociclib concentration range of 0.3 to $600 \mathrm{ng} / \mathrm{ml}$. The CC (calibration curve) samples were prepared and processed by spiking human plasma. To produce the best fit for the concentration/response relationship, a regression equation with a weighing factor $1 /$ (concentration ratio) 2 of the drug to internal normal concentration was used to create the calibration curve. The $r^{2}$ (coefficient of correlation) should be less than 0.98 as an acceptance criterion for linearity.

\section{Matrix selectivity and specificity}

Palbociclib's matrix selectivity was tested by analysing plasma from six separate lots, including one haemolytic and one lipemic plasma, to see if the analytes' and internal standard's retention times interfered. The intervention at the drug retention times was assessed by comparing the response in blank plasma to the response of LLOQ. The intervention at the internal standard's retention time was also compared to the response of the extracted internal standard in the LLOQ study. The interfering substance's response will be considered appropriate if it is less than $20 \%$ of the mean drug response in the LLOQ sample and less than $5 \%$ in the internal norm. 


\section{Recovery study}

This study was performed at three concentration levels: LQC, MQC-1, and HQC. The specimens, both extracted and non-extracted, have been packed. Internal criteria were applied at all stages, and samples were processed and injected. LQC, MQC-1, and HQC samples were freshly prepared for the preparation of extracted samples; internal criteria were applied at all stages, and samples were processed and injected and percent of recovery was measured separately for analytes and internal standard. It was determined what the average total recovery was.

\section{Dilution integrity study}

To test the dilution integrity analysis of the established palbociclib process, 12 sets of DIQC samples of palbociclib were prepared at $600 \mathrm{ng} / \mathrm{ml}$. Six sets of samples were diluted twice, and another six sets of samples were diluted four times. These palbociclib samples were analysed and concentrations were calculated by multiplying the concentration by 2 in the case of a 2 times dilution and multiplying by 4 in the case of a 4 time dilution.

\section{Ruggedness study}

To assess the robustness of the established palbociclib method, one accuracy and accuracy batch of samples was prepared and performed. These were injected into the LC-MS/MS system after being processed. To prepare the sample and mobile phase, different batch reagents (acetonitrile, ammonium format, HPLC grade water) were used, as well as the same type of other column with the same make on different instruments.

\section{Stability studies}

Along with room temperature and refrigerator stock solution stability study of palbociclib other stability tests of palbociclib (Bench top, wet extract, freeze thaw, autosampler, short term and long term stability) were performed using freshly prepared calibration curve samples and quality control samples at medium, middle, and high levels and analysed. The concentration of stability samples was calculated using data from concentration response linearity. Concentration response linearity data was collected and used to calculate the concentration of stability samples.

\section{Room temperature stability study}

It was conducted with a palbociclib stock solution that had been ready for at least 6 hours. Analyte stock solution and internal standard were prepared from source. The stock solution (stability samples) and fresh stock solution (comparison sample) were diluted to their final concentrations, which is equivalent to the final middle quality control analytes and internal norm. The percent of stability was measured after six replicates of fresh and comparison samples were injected immediately.

\section{Refrigerator stock solution stability}

To test palbociclib's stability, six replicates of the stock solution were prepared and stored in the refrigerator at $2-8^{\circ} \mathrm{C}$ for four days. On the day of the evaluation, a fresh reference stock solution (comparison sample) was prepared that was equal to the final MQC concentration of the analytes in reconstituted solution with the final concentration of the internal standard. Both stability and contrast samples were injected right away. For the analytes and internal normal, the percentage of stability for palbociclib was determined.

\section{Bench top stability}

Six sets of LQC and HQC palbociclib samples were taken from the deep freezer and left for $12 \mathrm{hr}$ unprocessed. Following that, six sets of new quality control samples (low, middle, and high) as well as calibration samples were prepared. Palbociclib stability samples were processed and analysed alongside fresh samples. The linearity data was used to measure the concentration.

\section{Autosampler stability}

To determine type stability, six sets of palbociclib quality control samples were prepared at the LQC and HQC levels and kept in an autosampler for three days. All stability samples were compared to freshly prepared spiked calibration curves and quality control samples at the low, middle, and high levels.

\section{Freeze thaw stability}

Four freeze-thaw cycles were used to determine it. Palbociclib LQC and HQC samples were prepared in six replicates and stored in a deep freezer at $-70^{\circ} \mathrm{C}$. The first six samples were removed after $24 \mathrm{hr}$ and thawed at room temperature before being frozen again. Similarly, the remaining samples were removed after the next $12 \mathrm{hr}$, and refrozen for another $12 \mathrm{hr}$. After four cycles, all samples were processed. Palbociclib stability samples were quantified at medium, mid, and high levels alongside freshly spiked calibration samples and quality control samples.

\section{Wet extract stability}

Six replicates of LQC and HQC samples were prepared, analysed, and held at room temperature $\left(20 \pm 5^{\circ} \mathrm{C}\right)$ for one day to investigate palbociclib wet extract stability. The samples were injected with freshly spiked calibration curve samples and quality control samples at medium, middle, and high levels after a suitable stability time. In comparison to freshly prepared samples, the sum of analytes in stability samples was measured.

\section{Short term stability at $-20^{\circ} \mathrm{C}$}

After spiking, six sets of palbociclib quality control samples at low and high levels were prepared and frozen at $-20^{\circ} \mathrm{C}$ in a deep freezer. The samples were processed three days later on the day of the evaluation, along with freshly prepared quality control samples at all stages and calibration curve samples. The stability samples' concentrations were measured in contrast to freshly prepared samples.

\section{Long term stability at $-70^{\circ} \mathrm{C}$}

Palbocilcib LQC and HQC samples were held at $-70^{\circ} \mathrm{C}$ for 30 days to examine this. Six sets of long-term quality control samples (LQC and HQC) were removed and processed with freshly prepared calibration curves and quality control samples on the day of the assessment. The calibration curve data was used to quantify all of the stability samples. All stability samples must have a mean percent nominal concentration of between 85 and 115 percent at each quality control level, with precision of less than $15 \%$ of the CV percent. At least $67 \%$ of the stability QC samples must be within $15 \%$ of their nominal values.

\section{Pharmacokinetic study}

The in vivo experimental research was carried out in compliance with the local ethical committee on animal experimentation's guidelines. The kinetic testing of palbociclib was conducted on three healthy rabbits weighing $1.87 \mathrm{~kg}, 2.07 \mathrm{~kg}$, and $2.13 \mathrm{~kg}$ and the study protocol was approved by the animal ethical committee no (CPCSEA regd. no.: 1287/ $\mathrm{PO} / \mathrm{Re} / \mathrm{S} / 09 / \mathrm{CPCSEA}$ and Approved proposal no.: IAEC/GIP-1287/ SM-S/Approved/11/2019-20). Palbociclib was given at a dose of $3.57 \mathrm{mg} / \mathrm{kg}$ through the oral route of administration. The dose measured for the animal form is the human dose, according to US FDA guidelines. ${ }^{15}$ The measured dose was administered as a suspension made by converting an equivalent amount of tablet powder. Every group received the suspension 
via a silicone rubber gastric intubation tube. Blood was drawn from the ear vein at $0,0.5,1,3,5,7,10$, and $24 \mathrm{hr}$ into polypropylene K3 EDTA coated tubes containing as an anti-coagulant. Plasma was collected and kept at $-20^{\circ} \mathrm{C}$ after centrifugation. The medication palbociclib was removed from plasma samples and placed into an autosampler tube for further analysis, according to the sample preparation methodology employed during method development. For each hour of blood collection, the drug concentration was assessed. PK Solver 2 is an application that allows you to solve problems quickly (a menu-driven adding programme for MS excel). ${ }^{16}$ After acquiring medication concentrations at various hours of blood sample collection, a time vs. concentration graph was drawn, and pharmacokinetic characteristics were calculated.

\section{RESULTS}

\section{Mass spectrometry}

The initial LCMS-MS was specified by proper tuning of all parameters in order to develop the method. The parameters were listed in detail in Table 1. Palbociclib and internal standard ribociclib created a simple parent ion in positive ion mode. Palbociclib was found to have a $\mathrm{m} / \mathrm{z}$ of 447.5 while ribociclib had a $\mathrm{m} / \mathrm{z}$ of 322.7 . The parent ion in the Q1 segment was protonated palbociclib, and the internal standard $[\mathrm{M}+\mathrm{H}]^{+}$ ion was used as a precursor ion to obtain Q3 product ion spectra.

\section{Method development}

Several chromatographic trials were carried out to develop the method, utilising different mobile phases with varied volume ratios. During the initial studies, several combinations of acetonitrile, methanol, and buffers were utilised. During the early experiments, the observed peaks of the analyte palbociclib were similarly unsatisfactory due to a large number of splits and a high amount of base line noise. Finally, a waters symmetry shield, $\mathrm{C}_{18}(4.6 \mathrm{~mm}$ id $\mathrm{x} 50 \mathrm{~mm})$ analytical column (waters Milford, MA, USA) with a mobile phase of Ultrapure water ( $\mathrm{PH} 4.2$, 0.2 percent acetic acid) and methanol $(50: 50) \mathrm{v} / \mathrm{v}$ was used. Even at very low quality control samples, the peak shape for analyte and internal standard was determined to be excellent in this optimised setting. To find a good internal standard, numerous compounds were explored. Finally,

Table 1: LC-MS/MS tuning parameters (Positive ion mode).

\begin{tabular}{ccc}
\hline Parameters & Palbociclib & $\begin{array}{c}\text { Internal standard } \\
\text { (Ribociclib) }\end{array}$ \\
\hline MRM & $\begin{array}{c}\mathrm{m} / \mathbf{z}+447.5 \\
\text { (parent) }\end{array}$ & $\begin{array}{c}\mathrm{m} / \mathrm{z}+434.5 \\
\text { (parent) }\end{array}$ \\
\cline { 2 - 3 } & $\begin{array}{c}\mathrm{m} / \mathrm{z}+380.3 \\
\text { (product) }\end{array}$ & $\begin{array}{c}\mathrm{m} / \mathrm{z} 322.7 \\
\text { (product) }\end{array}$ \\
\hline Declustaring Potential DP (V) & 72 & 72 \\
Collision energy CE (V) & 38 & 48 \\
Collision exit cell potential (V) & 26 & 32 \\
Source temperature & $382^{\circ} \mathrm{C}$ & $382^{\circ} \mathrm{C}$ \\
Curtain gas & $12 \mathrm{PSI}$ & $12 \mathrm{PSI}$ \\
Focusing potential & $350 \mathrm{~V}$ & $350 \mathrm{~V}$ \\
Dwell scan & $150 \mathrm{~ms}$ & $150 \mathrm{~ms}$ \\
Entrance potential & $10 \mathrm{~V}$ & $10 \mathrm{~V}$ \\
Ion spray voltage & $5000 \mathrm{~V}$ & $4500 \mathrm{~V}$ \\
Desolvation temperature & $700^{\circ} \mathrm{C}$ & $700^{\circ} \mathrm{C}$ \\
Desolvation gas flow $(\mathrm{L} / \mathrm{h})$ & 73 & 73 \\
\hline
\end{tabular}

$\mathrm{MRM}=$ multiple reaction monitoring
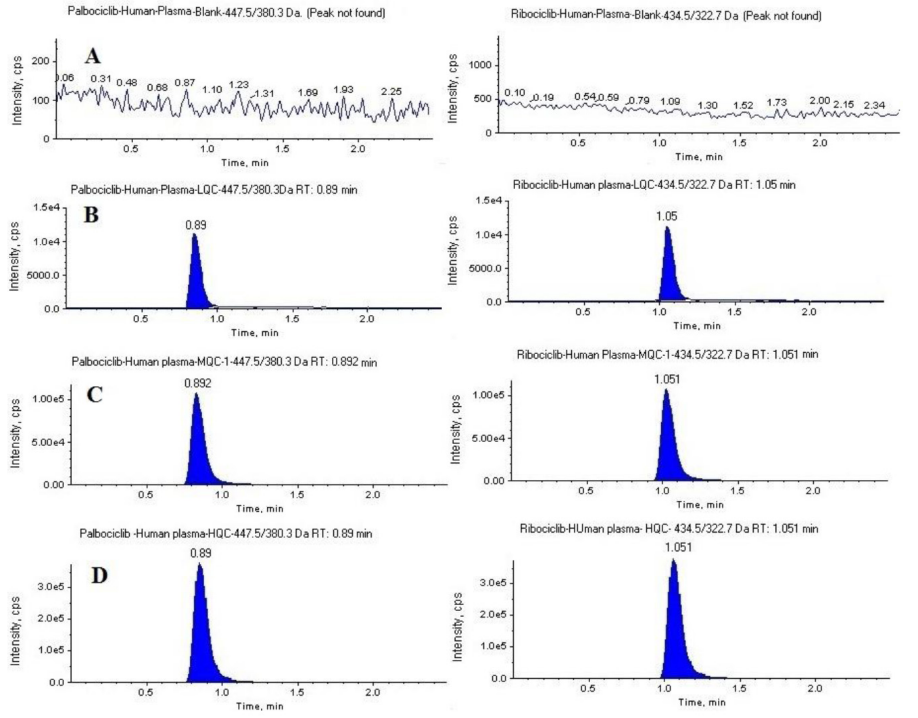

Figure 2: MRM chromatograms of palbociclib, Blank plasma (A), LOQ samples with internal standard [left panel] (B), MQC-1 samples with internal Standard [right panel] (C), HQC samples with internal Standard [right panel] (D).

ribociclib was chosen since the retention time and other values were very selective and did not interfere with analytes. The retention time of palbociclib was found to be 0.892 minutes at this optimised setting, and the retention time of the internal standard was found to be $1.051 \mathrm{~min}$, as shown in the MRM chromatograms in Figure 2.

\section{Validation}

Using the developed improved approach, several validation parameters according to USP requirements were investigated for palbociclib. The results of the carryover research reveal that at the ULOQ and LLOQ levels, there was no interference in the retention period of palbociclib and the internal standard ribociclib. The obtained answer for blank samples is 0 and the computed percent carry over is also 0 percent, indicating that the acceptance conditions were met. The internal standard normalised factor for the analyte in the presence of matrix ion was determined as a result of the matrix effect. Acceptance criteria were met when the percent $\mathrm{CV}$ of the normalised factor was found to be $1.58 \%$ for LQC samples and $0.63 \%$ for HQC samples. When comparing the mean response of extracted LLOQ samples with the blank matrix, no interference was discovered at the retention time of analyte and internal standard, and the response of interfering peaks at the retention time of analyte and internal standard was found to be $0 \%$ of the mean drug response. The intraday within batch precision (\% CV) of LLOQ, LQC, MQC-I, MQC-II, and HQC samples of palbociclib were reported to be $1.90,1.38,1.31,0.75$, and 1.45 , respectively, in a study of intraday within batch precision (\% CV). LLOQ, LQC, MQC-I, MQC-II, and HQC had intraday accuracy of $96.66 \%, 97.80 \%, 98.79 \%, 98.23 \%$, and $97.27 \%$, respectively. The batch accuracy (\% Nominal) results for the levels of LLOQ, LQC, MQC-I, MQC-II, and HQC were in the range of 92.94 percent to $98.35 \%$, and precision (\% CV) values were in the range of 0.76 to 3.58. Table 2 summarizes the results. The linearity investigation used a regression equation with a weight factor of 1 / (concentration ratio) 2 of medicines to internal standard concentration for palbociclib calibration curve samples in the concentration range of 0.3 to $600 \mathrm{ng} / \mathrm{ml}$. The correlation coefficient $\left(r^{2}\right)$ for palbociclib was determined to be 0.998 . In a recovery analysis of palbociclib and internal standard QC samples, 
Table 2: Accuracy and precision for determination of palbociclib in human plasma.

\begin{tabular}{|c|c|c|c|c|c|}
\hline \multirow[t]{2}{*}{ QC levels } & \multicolumn{5}{|c|}{ Palbociclib measured concentration $(\mathrm{ng} / \mathrm{ml})$} \\
\hline & Run* & Mean & SD & $\% \mathrm{CV}$ & $\%$ Nominal \\
\hline \multirow{6}{*}{ LLOQ } & \multicolumn{5}{|c|}{ Between the Batch Intra day } \\
\hline & 1 & 0.29 & 0.01 & 2.01 & 95.05 \\
\hline & 2 & 0.28 & 0.03 & 2.80 & 94.22 \\
\hline & 3 & 0.28 & 0.01 & 3.58 & 92.94 \\
\hline & 4 & 0.28 & 0.04 & 4.99 & 78.43 \\
\hline & 1 & 9.76 & 0.13 & 1.33 & 97.65 \\
\hline \multirow{2}{*}{ LQC } & 2 & 9.58 & 0.16 & 1.68 & 80.83 \\
\hline & 3 & 9.70 & 0.18 & 1.81 & 97.05 \\
\hline \multirow{6}{*}{ MQC-1 } & 4 & 0.75 & 0.14 & 1.44 & 97.50 \\
\hline & 1 & 48.94 & 0.79 & 1.61 & 97.87 \\
\hline & 2 & 49.20 & 1.06 & 2.16 & 49.02 \\
\hline & 3 & 49.05 & 1.44 & 2.32 & 96.44 \\
\hline & 4 & 49.20 & 1.06 & 2.16 & 98.04 \\
\hline & 1 & 98.35 & 1.17 & 1.19 & 98.35 \\
\hline \multirow{2}{*}{ MQC-II } & 2 & 98.17 & 0.74 & 0.76 & 98.17 \\
\hline & 3 & 98.35 & 1.17 & 1.19 & 98.35 \\
\hline \multirow{6}{*}{ HQC } & 4 & 98.30 & 0.79 & 0.80 & 98.30 \\
\hline & 1 & 291.74 & 4.29 & 1.47 & 97.24 \\
\hline & 2 & 289.63 & 8.24 & 2.84 & 96.26 \\
\hline & 3 & 289.46 & 7.78 & 2.69 & 96.49 \\
\hline & 4 & 271.28 & 23.8 & 8.77 & 92.92 \\
\hline & \multicolumn{5}{|c|}{ Within batch Interday } \\
\hline LLOQ & & 0.029 & 0.01 & 1.90 & 96.66 \\
\hline LQC & & 9.78 & 0.14 & 1.38 & 97.80 \\
\hline MQC-1 & & 49.40 & 0.65 & 1.31 & 98.79 \\
\hline MQC-II & & 98.23 & 0.74 & 0.75 & 98.23 \\
\hline HQC & & 291.82 & 4.24 & 1.45 & 97.27 \\
\hline
\end{tabular}

*each run includes six replicates.

the mean overall recovery of palbociclib was $95.96 \%$ and $94.07 \%$ for internal standard, with a precision (percent CV) range of 0.65 to 1.8 and a percent difference between height and lowest percent recovery of 7.26 for palbociclib and 6.92 for ribociclib, respectively. The results were within acceptable bounds. Palbociclib's dilution integrity was measured at the ULOQ level in this investigation. In comparison to the undiluted calibration curve samples, \% CV and percent nominal were found to be 1.16 and $96.92 \%$ at two times dilution and 1.36 and $96.10 \%$ at four times dilution. Within batch precision (\% CV) was reported to be $2.15,1.54$, $0.97,0.57$, and 5.91 for the ruggedness investigation. At the LLOQ, LQC, MQC-I, MQC-II, and HQC levels, the accuracy values were $96.44 \%$, $96.43 \%, 98.35 \%, 98.51 \%$, and $92.37 \%$, respectively.

\section{Stability studies}

The percent stability for palbociclib and the internal standard were $101.33 \%$ and $98.43 \%$, respectively, according to the results of a room temperature $\left(20 \pm 5^{\circ} \mathrm{C}\right)$ stock solution stability analysis. The calculated percent of stability for palbociclib and 99.29 for the internal standard was found in a refrigerator stock solution stability study at $2-8^{\circ} \mathrm{C}$ for 4 days. At the LQC level, the percent stability for Bench top stability research results was $98.64 \%$, and at the HQC level, it was $99.39 \%$. The autosampler stability research $(72 \mathrm{hr}$ of acceptable stability period in autosampler) revealed $102.74 \%$ and $100.57 \%$ at the LQC and HQC levels, respectively. Palbociclib has a wide range of acceptability at both the LQC and HQC levels, with percentage stability of 102.17 percent and 99.22 percent, respectively, according to the results of a four-cycle freezethaw stability sample. In wet extract stability testing, the percent nominal value for palbociclib was 94 percent for LQC and 93.68 percent for HQC. Palbociclib's computed percent stability was more than $102.06 \%$ at LQC and $98.63 \%$ at HQC, which met the short-term stability study's acceptability level. The percentage stability for LQC samples was $101.99 \%$ and $98.49 \%$ for HQC samples in the long-term stability testing (30 days at $-70^{\circ} \mathrm{C}$ ). The findings of all stability investigations are summarised in Table 3.

\section{Result of pharmacokinetic study}

The non-compartmental model was used to compute the pharmacokinetic parameters of the kinetic research, and the area under the curve $\left(\mathrm{AUC}_{0}-\infty\right)$ for palbociclib was $2632 \pm 6.98 \mathrm{hr} . \mathrm{ng} / \mathrm{ml}$. The half-life of elimination $(\mathrm{t} 1 / 2)$ is $11.3 \pm 5.3 \mathrm{hr}$. The Table 4 of kinetic parameters lists all of the different parameters in detail. Figure 3 depicted the plasma concentration as well as the temporal profile. Figure 4 shows the MRM chromatograms of palbociclib in rabbit blood samples during the kinetic investigation.

\section{DISCUSSION}

The present bioanalytical method for palbociclib has been developed after successful trials to optimised the parameters for both chromatographic and mass spectrophotometric analysis. Initially the LCMS parameters has been tuned to obtained the product ion mass spectra of palbociclib and internal standard Ribociclib. Using the same tuned condition MRM chromatograms of palbociclib and Ribociclib has been optimized using methanol and ultrapure water, $\mathrm{pH} 4.2$ in the volume ratio of 60:40. The obtained MRM chromatograms of both palbociclib and internal standard was highly selective with excellent peak shape with great sensitivity. The results of all the validation parameters are within the acceptance criteria as per US-FDA bio analytical method development guidelines. ${ }^{17}$ The result of carry over test shows $0 \%$ carryover of LLOQ and ULOQ samples of analyte and internal standard which satisfied the acceptance criteria. The results of the matrix and analyte selectivity analysis revealed that the palbociclib developed method is selective

Table 3: Stability study data of palbociclib.

\begin{tabular}{cccccc}
\hline \multirow{2}{*}{ QC levels } & Type of & \multicolumn{4}{c}{ Palbociclib } \\
\cline { 3 - 6 } stability & Mean* & SD & \%CV & \%Nominal \\
\hline \multirow{4}{*}{ LQC } & Bench Top & 9.07 & 0.17 & 1.89 & 90.68 \\
& Freeze thaw & 9.39 & 0.23 & 2.14 & 93.90 \\
& Autosampler & 9.32 & 0.29 & 3.10 & 93.17 \\
& Wet extract & 9.40 & 0.24 & 2.58 & 94.00 \\
& Short term & 9.38 & 0.20 & 2.11 & 93.80 \\
& Long term & 9.37 & 0.14 & 1.48 & 93.70 \\
& Bench Top & 281.34 & 7.43 & 2.64 & 93.78 \\
& Freeze thaw & 281.09 & 4.99 & 1.77 & 93.69 \\
& Autosampler & 281.33 & 3.96 & 1.41 & 94.26 \\
& Wet extract & 281.07 & 4.98 & 1.77 & 93.69 \\
& Short term & 279.41 & 4.96 & 1.78 & 93.13 \\
& Long term & 279.04 & 4.23 & 1.52 & 93.01 \\
\hline
\end{tabular}

*mean of six replicates 
Table 4: Pharmacokinetic parameters of Palbociclib.

\begin{tabular}{cc}
\hline Pharmacokinetic parameters & Palbociclib \\
\hline $\left.\mathrm{C}_{\max (} \mathrm{ng} / \mathrm{ml}\right)$ & $115.2 \pm 0.18$ \\
$\mathrm{t}_{\max }$ & $7 \mathrm{hr} \pm 1.39$ \\
$\mathrm{AUC}_{0-\infty}\left(\mathrm{h}^{*} \mathrm{ng} / \mathrm{ml}\right)$ & $2632 \pm 6.98$ \\
$\mathrm{AUC}_{0-\mathrm{t}}\left(\mathrm{h}^{*} \mathrm{ng} / \mathrm{ml}\right)$ & $2789 \pm 7.36$ \\
$\mathrm{t}_{1 / 2}(\mathrm{~h})$ & $11.3 \pm 5.3$ \\
\hline
\end{tabular}

AUC (Area under the curve),

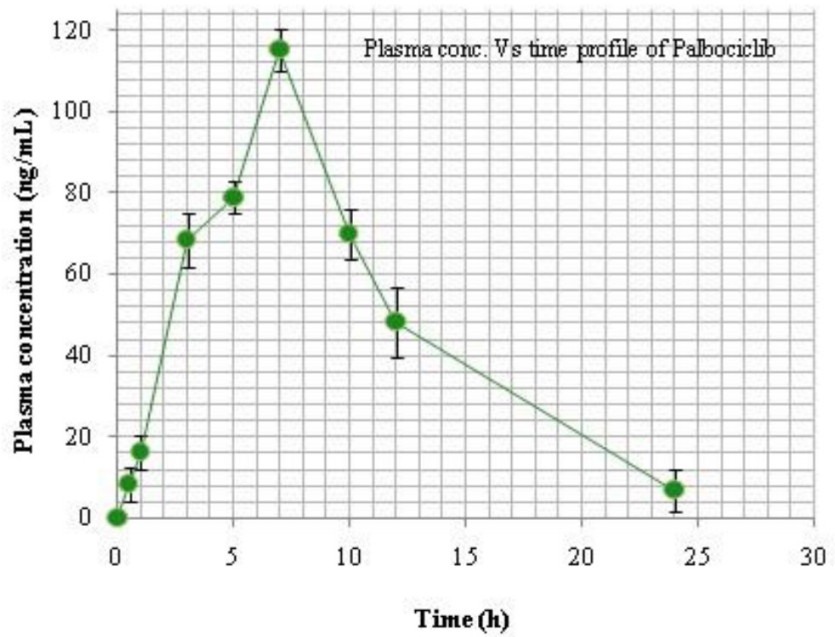

Figure 3: Mean plasma concentration -time profile of palbociclib after oral administration of palbociclib tablet at human equivalent dose.
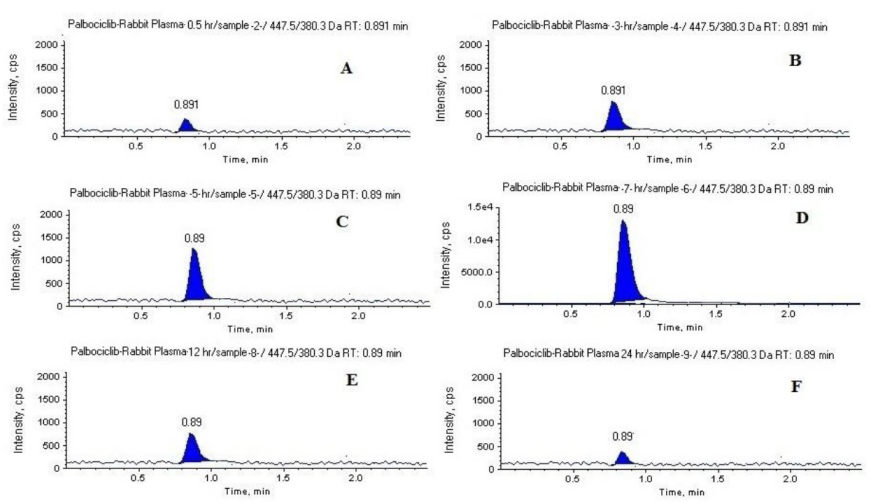

Figure 4: MRM Chromatograms of palbociclib within rabbit plasma sample for kinetic study at 0.5 hour (A), $3^{\text {rd }} \mathrm{hr}(\mathrm{B}), 5^{\text {th }} \mathrm{hr}$ (C) $7^{\text {th }} \mathrm{hr}(\mathrm{D}), 12^{\text {th }} \mathrm{hr}$ (E) $24^{\text {th }} \mathrm{hr}$ (F) blood sample.

since there is no interference. The "IS normalised matrix factors" value in the matrix impact investigation is good, indicating that there was no significant matrix ion effect for palbociclib and internal standard. The findings of an intra- and interday precision and accuracy analysis at all quality control levels show that the \% CV values and percent nominal values were both within acceptable limits, indicating that the current procedure is highly precise and accurate. The regression equation and linearity graph demonstrated a best fit relationship with a correlation coefficient $\left(r^{2}\right)=0.998$ for palbociclib in the linearity research findings employing calibration curve samples in the range of 3-600 ng/ml, demonstrating the linearity of the developed technique via LC-MS/MS. In the LQC, MQC-1, and HQC levels, the mean overall recovery results of palbociclib and internal standards were found to be well satisfactory, and the percent recovery differences were within acceptable guidelines. The precision (percent CV) and accuracy (percent Nominal) values of 2 and 4 times dilution during the dilution integrity study were within acceptable limits. The results of the dilution integrity study indicate that the quality of the analyte is unaffected by sample dilution, indicating that the sample has integrity over dilution. The accuracy and precision values in the ruggedness study showed satisfactory accuracy and precision values in all QC levels that were within the limits, indicating the method's ruggedness when changing columns and reagents. The calculated percent of stability values in stock solution stability at room temperature $\left(25^{\circ} \mathrm{C}\right)$ and in the refrigerator $\left(2-8^{\circ} \mathrm{C}, 4\right.$ days $)$ were found to be satisfactory and within the range, indicating that the developed method is stable. Stability samples were compared with freshly prepared samples in bench top, wet extract, freeze thaw, autosampler, short and long term $\left(-70^{\circ} \mathrm{C}, 30\right.$ days $)$ stability studies, and concentration was back calculated from the calibration curve samples. In category of stability study the mean $\%$ of nominal values were found within $94.53 \%$ to $100.79 \%$ and $\% \mathrm{CV}$ values were within $10 \%$, which strongly demonstrated about the stability of all quality control samples and stability of the developed method. During the analysis of pharmacokinetic samples, the newly developed and validated palbociclib method performed admirably. All parameters can be calculated because the plasma concentration and time are smooth. One compartmental model fit all of the kinetic parameters well. The present method's selectivity, sensitivity, and specificity were sufficient for determining the pharmacokinetic profile of palbociclib in rabbit plasma samples.

\section{CONCLUSION}

The current method has a higher throughput than the previously published HPLC method. With less run time, the total analysis time is required to be much less. This method became more practical and cost effective thanks to a simple liquid-liquid extraction procedure. Empirical evidence of all validation results showed that the method is highly validated and simple, as all parameters are within the US-FDA guidelines' acceptance limits. As a result, this current attractive, simple, and reliable novel method is unquestionably highly applicable for palbociclib quantitative analysis during clinical trials, preclinical trials, forensic, and toxicological studies.

\section{ACKNOWLEDGEMENT}

The authors are thankful to the management of GITAM Institute of Pharmacy, Visakhapatnam, for providing the facilities for this work

\section{CONFLICT OF INTEREST}

The authors declare that there is no conflict of interest.

\section{ABBREVIATIONS}

LC-MS/MS: (Liquid chromatography tandem mass spectroscopy); BC: Breast cancer; MBC: Metastatic breast cancer; CBR: Clinical beneficiary rate; HER2: Human epidermal growth receptor; MRM: Multiple reaction monitoring; CV: Coefficient of variation; RSD: Relative standard deviation; AUC: Area under the curve; USFDA: United State food and drug administration; HPLC: High performance liquid chromatography; HQC: Higher quality control; MQC: Medium quality control; LQC: Lower quality control; LLOQ: Lower limit of quantitation; DIQC: Dilution integrity quality control. 


\section{REFERENCES}

1. Cardoso F, Spence D, Mertz S, Corneliussen-James DC, Sabelko K, Gralow J, et al. Global analysis of advanced/metastatic breast cancer: decade report (2005-2015). Breast. 2018;39:131-8. doi: 10.1016/j.breast.2018.03.002, PMID 29679849

2. Tripathy D, Im SA, Colleoni M, Franke F, Bardia A, Harbeck N, et al. Ribociclib plus endocrine therapy for premenopausal women with hormone-receptorpositive, advanced breast cancer (MONALEESA-7): A randomised phase 3 trial. Lancet Oncol. 2018;19(7):904-15. doi: 10.1016/S1470-2045(18)30292-4, PMID 29804902.

3. Beaver JA, Amiri-Kordestani L, Charlab R, Chen W, Palmby T, Tilley A, et al. FDA approval: Palbociclib for the treatment of postmenopausal patients with estrogen receptor-positive, HER2-negative metastatic breast cancer. Clin Cancer Res. 2015:21(21):4760-6. doi: 10.1158/1078-0432.CCR-15-1185, PMID 26324739.

4. Harper JW, Adams PD. Cyclin-dependent kinases. Chem Rev. 2001;101 (8):2511-26. doi: 10.1021/cr0001030, PMID 11749386

5. Samson K. LEE011 CDK inhibitor showing early promise in drug-resistant cancers. Oncology Times. Oncology Times. 2014;36(3):39-40. doi: 10.1097/01. COT.0000444043.33304.c1.

6. Nithiyananthan K, Prasadarao KVS. Analytical method development and validation of palbociclib in its pure dosage forms. J Pharm Biomed Anal Let. 2020:8(1):26-9.

7. Dange Y, Bhinge S, Salunkhe V. Optimization and validation of RP-HPLC method for simultaneous estimation of palbociclib and letrozole. Toxicol Mech Methods. 2018;28(3):187-94. doi: 10.1080/15376516.2017.1388458, PMID 28980851.

8. Sreelakshmi Ma SRLC, Raviteja BB. Simultaneous estimation of ribociclib and palbociclib in bulk samples by reverse phase high-performance liquid chromatography. Int J Pharm Biol Sci. 2019;9(2):413-21.

9. Kallepalli P, Annapurna MM. New stability-indicating liquid chromatographic method for determination of palbociclib (an anti-breast cancer drug). Int J Green Pharm. 2018;12(1):S270.
10. Posocco B, Buzzo M, Poetto AS, Orleni M, Gagno S, Zanchetta M, et al Simultaneous quantification of palbociclib, ribociclib and letrozole in human plasma by a new LC-MS/MS method for clinical application. PLOS ONE. 2020;15(2):e0228822. doi: 10.1371/journal.pone.0228822, PMID 32032379.

11. Martínez-Chávez AM, Rosing $H$, Hillebrand $M$, Tibben $M$, Schinkel $A H$, Beijnen $\mathrm{JH}$. Development and validation of a bioanalytical method for the quantification of the CDK4/6 inhibitors abemaciclib, palbociclib, and ribociclib in human and mouse matrices using liquid chromatography-tandem mass spectrometry. Anal Bioanal Chem. 2019;411(20):5331-45. doi: 10.1007/s00216019-01932-W, PMID 31209549.

12. Jolibois J, Schmitt A, Royer B. A simple and fast LC-MS/MS method for the routine measurement of cabozantinib, olaparib, palbociclib, pazopanib, sorafenib, sunitinib and its main active metabolite in human plasma. J Chromatogr B Analyt Technol Biomed Life Sci. 2019;1132:121844. doi: 10.1016/j. jchromb.2019.121844, PMID 31678788.

13. Nalanda RB, Rao AS, Sankar DG. Determination of palbociclib in human plasma using high-performance liquid chromatography - ultraviolet detection. Int J Pharm Sci Res. 2018;9(9):3883-88.

14. US Food and Drug Administration. Guidance for industry, bioanalytical method validation; 2013

15. US FDA guidelines for equivalent dose calculation.

16. Zhang Y, Huo M, Zhou J, Xie S. PK Solver: An add-in program for pharmacokinetic and pharmacodynamic data analysis in Microsoft Excel. Comput Methods Programs Biomed. 2010;99(3):306-14. doi: 10.1016/j.cmpb.2010.01.007, PMID 20176408.

17. Prasenjit M, Satla S, Raparla R. Quantification of Blonanserin in human plasma using liquid chromatography- electrospray ionization-tandem mass spectrophotometry-application to pharmacokinetic study. J Young Pharm 2016;8(4):406-14. doi: 10.5530/jyp.2016.4.17.

Article History: Received: 30-09-2021; Revised: 23-10-2021; Accepted: 03-12-2021

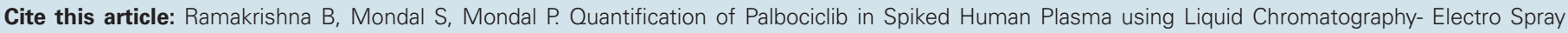
Ionization-tandem Mass Spectrophotometry- an Application to Pharmacokinetic Study. J Young Pharm. 2022;14(1):82-8. 